\title{
Follicular Changes In Relation To Menstrual Cycle In Female Of Fertile Age
}

\author{
Atif Ateeq ${ }^{1}$, Jayant Kumar Verma ${ }^{2}$,A.K.Gupta ${ }^{3}$, Nityanand Srivastava $^{4}$, \\ Vinod Kumar ${ }^{5}$,Arun Nagrath ${ }^{6}$, Anuj Jain ${ }^{7}$, \\ ${ }^{\text {IJR3 }}$ Department of Anatomy, UPUMS Saifai 2-Associate Professor, Department of Anatomy, UPUMS Saifai \\ ${ }^{3}$ Professor \& HOD, Department of Radiology, UPUMS Saifai \\ ${ }^{4}$ Professor \& HOD Department of Anatomy, UPUMS Saifai \\ ${ }^{5}$ Professor \& HOD, Department of Anatomy, Saraswati Medical College, Unnao, (U.P.), 6- Professor \& HOD, \\ Department of Obs \& Ganae, UPUMS Saifai \\ ${ }^{7}$ Professor, Department of Anatomy, UPUMS Saifai.
}

\begin{abstract}
Follicular changes studiedultrasonographically by Transabdominal as well as Transvaginal route during follicular monitoring in 81 patients of fertile age. These patients were referred for follicular monitoring in normal and stimulated cycles from infertility clinic. Patients' compliance and acceptance was excellent for TVS technique as compared to TAS technique. For follicles > $20 \mathrm{~mm}$ in size, no significant difference was noted in detection rate between TAS and TVS. However, in smaller follicles especially below $10 \mathrm{~mm}$ the TVS offered definite improvement in the detection rates. Transabdominal methods underestimates the number of follicles, than does TVS. TV ultrasound measurement of follicular size has lower intra and inter-observer variability. Other studies also demonstrated that TVS scanning methods have a higher degree of patients compliance and acceptance of TVS has already been confirmed in our study. This highlights the need to make use of this mode of investigation for wider indications.
\end{abstract}

Keywords: Follicle, Ovulation,Ultrasound,TAS,TVS

\section{Introduction}

The ovaries are ovum-producing, paired flattened ovoid organs of the female reproductive and endocrine systems. Each ovary is $2.5-3.5 \mathrm{~cm}$ long, $1.5 \mathrm{~cm}$ broad, and $1 \mathrm{~cm}$ thick in the multiparous women.They have an average volume of $12 \mathrm{~cm}^{3}$ in reproductive mature women. The reproductive system of women shows regular cyclic changes that physiogically may be regarded as periodic preparations for fertilization and contraception. ${ }^{7,9}$ At beginning of each ovarian cycle, 15 to 20 primary-stage (preantral) follicles are stimulated to grow under the influence of FSH. The ovarian cycle consists of the follicular phase, ovulation, and luteal phase whereas the uterine cycle is divided into menstrual phase, proliferative phase, and secretory phase. ${ }^{2}$ In the human ovary, 2 million oocytes are found at birth, and about 400,000 follicles are present at the onset of puberty. The remaining follicles are depleted at a rate of approximately 1000 follicles per month until 35 years of age, when this rate accelerates. Only 400 follicles are normally ovulated during female reproductive life. More than 99.9 percent of follicles undergo the degenerative process known as atresia through a process of cell death termed apoptosis.

All primordial follicles are composed of a small primary oocyte arrested in the diplotene stage of meiosis, a single layer of flattened (squamous) granulosa cells known as follicular cells and a basal lamina. ${ }^{8}$ Recruitment is the process by which an arrested primordial follicle is triggered to reinitiate development and enter the pool of growing follicles. As primordial follicles begin to grow and develop into a primary follicle.Some of these primary follicle die, while others begin to accumulate fluid (liquor folliculi) in a space called the antrum, thereby entering the antral or vesicular stage.Tertiary follicle Although a number of follicles may progress to the secondary stage by about the first week of a menstrual cycle, usually only one follicle, from either one of the two ovaries, proceeds to the tertiary stage, and the remainder become atretic. ${ }^{12}$ The surviving follicle increases in size considerably as the antrum takes up fluid from the surrounding tissues and enlarge to a diameter of $2 \mathrm{~cm}$. The cumulus oophorus surrounding the oocyte thins.

The term Graafian follicle is often used to describe this mature follicular stage. Ovulation is the process by which an ovum, in the form of secondary oocytes, is discharged from the ovary to become a gamete.Following ovulation, the corpus luteum develops from the remains of the dominant or Graffian follicle in a process referred to as luteinization. ${ }^{8,17}$ 


\section{Ovarian Follicular Monitoring}

Ultrasound is a key investigation in obstetrics and gynaecology and is commonly used in screening, diagnosing, treatment and follow-up. It is quick, cheap, widely available, portable and does not involve ionizing radiation.Ultrasound is an extension of the clinical examination in gynaecology.Ultrasound has made significant improvement in the management of infertility. It has a role in the initial assessment of ovulation, in monitoring of treatment cycles and the performance of ultrasound guided procedures. The use of ultrasonography to monitor follicular growth and confirm the occurrence of ovulation or ovulatory failure has clinically established along with well timed circulating hormone level, ultrasound offers answers to subtle problems in ovulatory dysfunction by the practicing clinician. ${ }^{4}$

\section{Material And Methods}

The study was conducted on fertile female patient in the Department of Anatomy with close collaboration of Department of Radiodiagnosis and Department of Obstetrics and Gynaecology, Uttar pradesh University of Medical Sciences, Saifai, Etawah. Verbal and written consent was obtained prior to perform ultrasound examination. Transabdominal ultrasound scanning always preceded transvaginal ultrasound scanning and in any given case the same sonologist interpreted both the scan. Transvaginal ultrasound has dramatically advanced the ability of the Sonologist to evaluate the monitoring gynecologic infertility patients. All the patients had distended urinary bladder during transabdominal ultrasound but transvaginal ultrasound scanning done empty bladder. A distended urinary bladder is essential to displace the bowel loops and to provide an acoustic window. After performing the transabdominal ultrasound all patients voided completely before the transvaginal ultrasound scanting. Transvaginal ultrasound was done in the same sitting immediately after transabdominal ultrasound.

The ovary were measured in three planes and the volume was calculated. The study was performed from Day 9 -17 of menstrual cycle. Serial monitoring of follicular development was done in both natural and stimulated ovarian cycles' The follicle appears as an echo free structure, $7-8 \mathrm{~mm}$ in size, around the ovarian tissue, which is more echgenic ${ }^{19}$

\section{Result And Observations}

The present study was conducted in the department of Anatomy, department of Obstetrics and Gynecology and department of radiodiagnosis. A total 81 married female patient were studied.

\section{Age Wise Distribution}

\begin{tabular}{|l|c|c|c|c|c|c|}
\hline & \multicolumn{2}{|c|}{ Frequency } & Mean & SD & t-value & p-value \\
\cline { 1 - 3 } Method & $\leq 30$ yrs & $>30$ yrs & & & & \\
\hline TAS & 25 & 21 & 29.9 & 3.72 & -1.1233 & 0.2653 \\
\hline TVS & 23 & 12 & 30.6 & 1.75 & & \\
\hline
\end{tabular}

The two methods are not significantly different.at 0.05 level of significance.

\section{AGE WISE DISTRIBUTION}

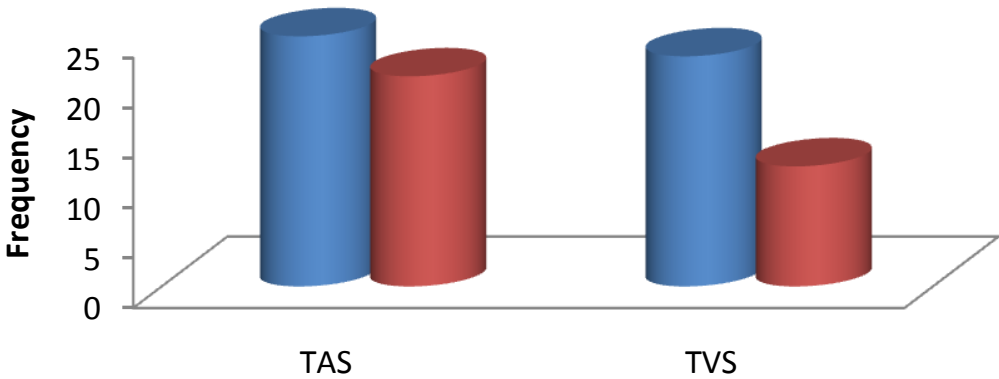

Method

Frequency $\leq 30$ yrs $\quad$ Frequency $>30$ yrs

Of the 81 patient $56.79 \%$ (46out of 81) patients were examined by Transabdominal (TAS) method and $43.20 \%$ (35out of 81) were examined by Transvaginal (TVS) method. 
Out of 46 patient those were examined by TAS, 25 patients were below the age of 30 years and 21 patients were under the age of 21 .Out of 35 patient examined by TVS, 23 patients were under the age of 30 years and 12 patients were below the age of 21 .

Table-1a Comparison Of Baseline Sizes Of Follicle (Ro). N = 81

\begin{tabular}{|c|c|c|c|c|}
\hline Method & Mean & SD & t-value & p-value \\
\hline TAS & 10.59 & 3.39 & \multirow{2}{*}{-0.7628} & 0.4483 \\
\hline TVS & 11.22 & 3.89 & & 0.48 \\
\hline
\end{tabular}

The two methods are not significantly different.at 0.05 level of significance

During TAS, the mean baseline size of follicle in right ovary was measured $10.59 \mathrm{~mm}$ with SD 3.39 and in TVS, it was measured $11.22 \mathrm{~mm}$ with SD 3.89. (Table 1A)

\section{COMPARISON OF BASELINE SIZES OF FOLLICLE}

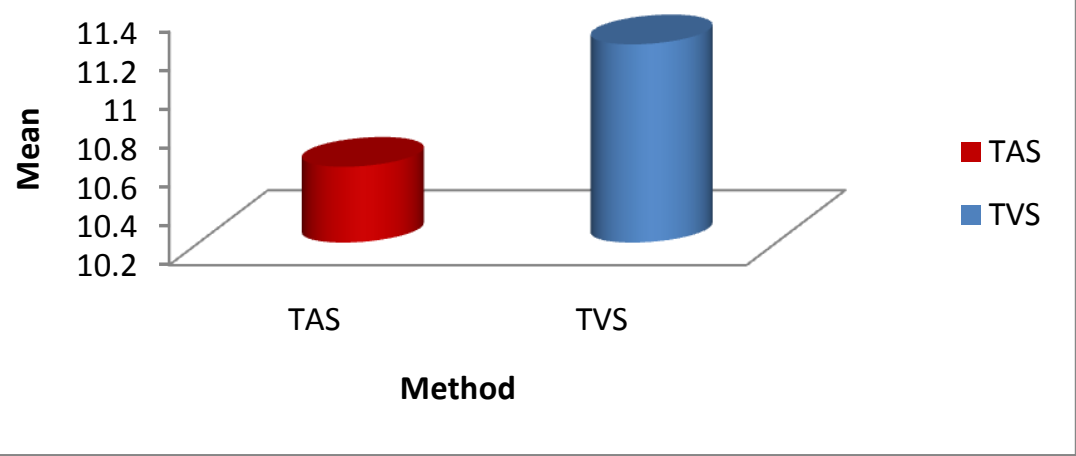

Table-1b

Comparison Of Baseline Sizes Of Follicle (Lo). $\mathbf{N}=81$

\begin{tabular}{|c|c|c|c|c|}
\hline Method & Mean & SD & t-value & p-value \\
\hline TAS & 10.88 & 4.17 & \multirow{2}{*}{-1.6447} & 0.1046 \\
\hline TVS & 12.48 & 4.46 & 0.76 \\
\hline
\end{tabular}

The two methods are not significantly different.at 0.05 level of significance While performing TASthe mean baseline size of follicle in left ovary was measured $10.88 \mathrm{~mm}$ with SD 4.17 and in TVS scanning it was measured $12.48 \mathrm{~mm}$ with SD 4.46. (Table 1B).

\section{COMPARISON OF BASELINE SIZES OF FOLLICLE}

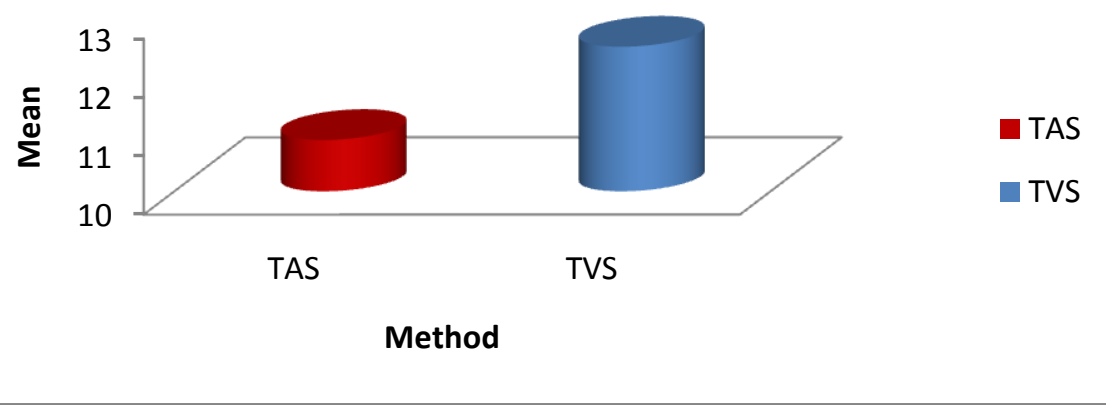


Table-2a

Comparison of number of dominant follicle (ro). $\mathrm{N}=81$

\begin{tabular}{|c|c|c|c|c|}
\hline Method & Average Number & SD & t-value & p-value \\
\hline TAS & 1.19 & 0.40 & \multirow{2}{*}{-1.7703} & \multirow{2}{*}{0.0814} \\
\hline TVS & 1.37 & 0.49 & \\
\hline
\end{tabular}

The two methods are not significantly different.at 0.05 level of significance During TAS, The average number of dominant follicle was found to be 1.19 with SD 0.40 in RO, while in case of TVS it was 1.37 with SD 0.49.(Table 2A)

\section{COMPARISON OF NUMBER OF DOMINANT FOLLICLE (RO)}

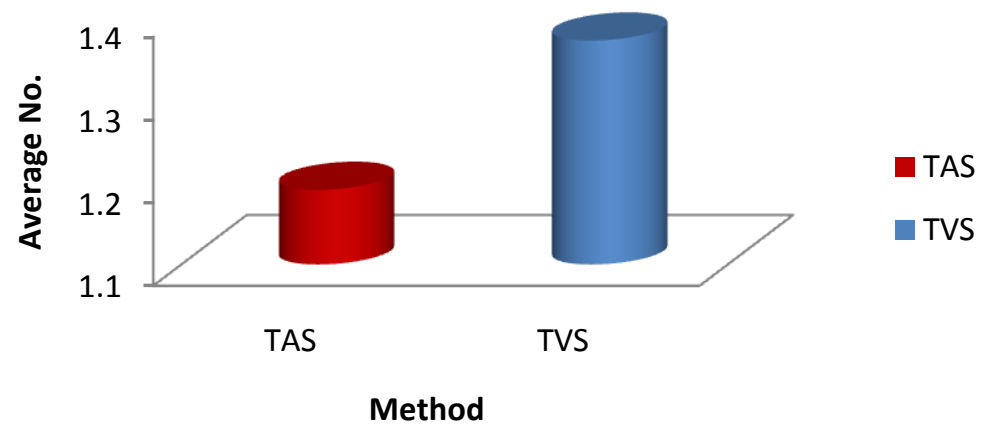


Table-2b Comparison of number of dominant follicle (lo). $\mathrm{N}=81$

\begin{tabular}{|c|c|c|c|c|}
\hline Method & Average Number & SD & t-value & p-value \\
\hline TAS & 1.20 & 0.42 & \multirow{2}{*}{-1.6741} & 0.099 \\
\hline TVS & 1.38 & 0.52 & \\
\hline
\end{tabular}

The two methods are not significantly different.at 0.05 level of significance During TAS, The average number of dominant follicle was found to be 1.20 with SD 0.42 in LO, while in case of TVS it was 1.38 with SD 0.52 (Table 2B).

\section{COMPARISON OF NUMBER OF DOMINANT FOLLICLE}

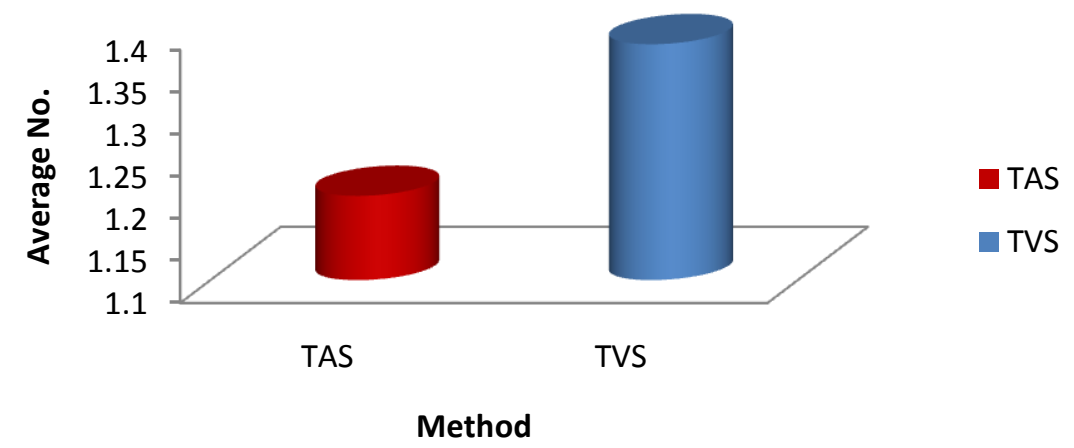

Table-3

\begin{tabular}{|c|c|c|c|c|}
\multicolumn{7}{|c}{ Comparison Of Endometrial Thickness, $\mathbf{N}=81$} \\
\cline { 1 - 3 } Method & $\begin{array}{c}\text { Average Endometrial } \\
\text { thickness }\end{array}$ & SD & t-value & p-value \\
\hline TAS & 7.56 & 0.81 & -9.8256 & $<0.0001$ \\
\hline TVS & 9.83 & 1.17 & -825 \\
\hline
\end{tabular}

The two methods are significantly different.at 0.05 level of significance Endometrial thickness as measured 7.56 mm with SD 0.81 on TAS while on TVS it was 9.83 with SD 1.17. (Table 3)

\section{COMPARISON OF ENDOMETRIAL THICKNESS}

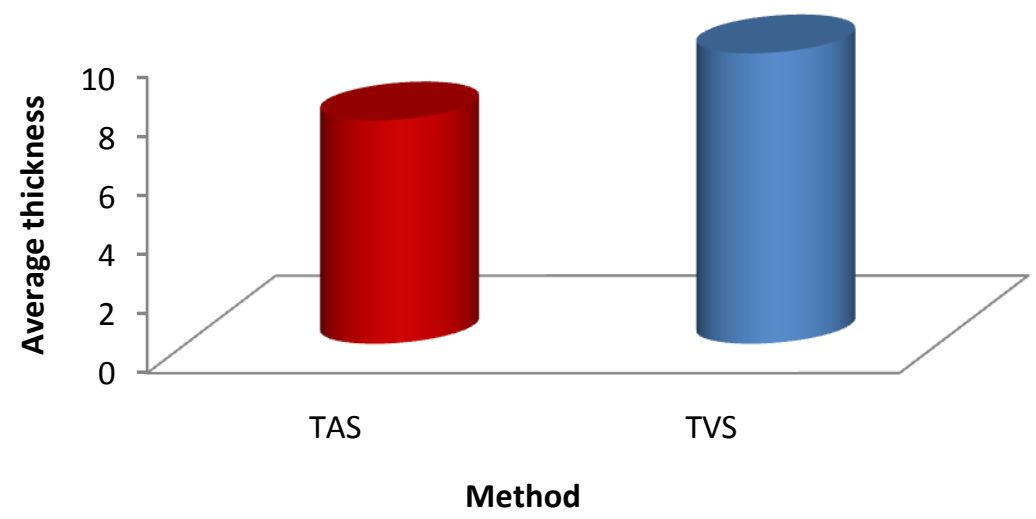




\section{Discussion}

In our study baseline sizes of the follicle in both ovaries by transvaginal and transabdominal ultrasound were compared. In present study it was observed that the mean baseline size of follicle in right ovary was 10.59 $\mathrm{mm}$ with SD 3.39 and in TVS, it was $11.22 \mathrm{~mm}$ with SD 3.89. (Table 1A) and in TAS the mean baseline size of follicle in left ovary was $10.88 \mathrm{~mm}$ with SD 4.17 and in TVS scanning it was $12.48 \mathrm{~mm}$ with SD 4.46. (Table 1B). By applying t-test, in right ovary value of t-test was -0.7628 and p-value was 0.4483 and the two data was found not significantly different at 0.05 level of signinificance and simlarly in left ovary the value of t-test was 1.6447 and p-value was 0.1046 and the two data was found not significantly different at 0.05 level of signinificance.

Ziban Nahar et al (2008) found no significant difference in follicles $>18 \mathrm{~mm}$ in size on comparing TAS and TVS. ${ }^{16}$

Farquhar et al (1994) did a comparative study between transabdominal and transvaginal route in the diagnosis of polycystic ovaries in 187 patients, and found no difference in the number of follicles or the size of the follicles. ${ }^{13}$

Mendelson EBet al (1988) concluded that transvaginal image quality was better in 79\%-87\% of scans; transabdominal image quality was better in 3\%-5\% of scans, in sonographic findings of 200 patients who underwent concurrent transabdominal and transvaginal pelvic ultrasound. In the present study the number of dominant follicle in each ovary by two method i.e. transvaginal and transabdominal. (Table 2A \& 2B)

During TAS, The average number of dominant follicle was found to be 1.19 with SD 0.40 in RO, while in case of TVS it was 1.37 with SD 0.49.(Table 2A) During TAS, the average number of dominant follicle was found to be 1.20 with SD 0.42 in LO, while in case of TVS it was 1.38 with SD 0.52 (Table 2B). In right ovary the value of t-test was -1.7703 with p-value 0.0814 and in left ovary these parameters were -1.6741 and 0.099 respectively. It was found the data was not significantly different at 0.05 level of significance. Andreotti RF et al (1989) concluded that transvaginal and transabdominal sonography (EVS and TAS, respectively) are effective methods for monitoring ovarian follicular development. In 41 sonographic studies they demonstrate that the number and size of dominant follicles correlated well with estradiol levels $(r=.9074)$ for EVS but less so $(r=.3816)$ for TAS. EVS has several advantages when monitoring follicular development. Baerwald AR et al (2003) compared dominant ovarian follicles of 18 clinically normal women using transabdominal and transvaginal ultrasonography for the 7 days before ovulation during a natural cycle $(n=9)$ or the 7 days before peak estradiol in women using OC $(n=11)$.No differences were observed in two other image, measured with either technique, between natural and OC cycle follicles. The endometrial thickness was measured i.e. TAS and TVS. On TAS the average endometrial thickness was 7.56 with SD 0.81 and on TVS he average endometrial thickness was 9.83 with SD 1.17. These two data were compared by applying t-test, t-value of which was 9.8256 and p-value was $<0.0001$ and the two data was found significantly different at 0.05 level of signinificance. (Table 9) Ghazi Sedeq et al (2016) found in theie comparative study on 100 women that Transvaginal ultrasound scanning is an excellent tool for the determination of whether further investigation with histopathological examination of endometrial biopsy is necessary or not for women presented with abnormal uterine bleeding. Transvaginal ultrasound sensitivity and specificity were $100 \%$ and $92.9 \%$, respectively while transabdominal ultrasound sensitivity and specificity were $92.8 \%$ and $65 \%$, respectively. ${ }^{14}$

Tsuda H et al (1995) conclude that TVS might be superior to TAS, especially in patients with a retroflexed uterus. The mean endometrial thickness estimated by TVS was larger than that obtained by TAS ( $\mathrm{p}<$ 0.0001 ). Sensitivity and specificity of TAS were 83.3 and $58.8 \%$ and of TVS 100 and $54.1 \%$, respectively. ${ }^{18}$

\section{Conclusion}

Transabdominal sonography (TAS) and Transvaginal sonography (TVS) were compared for follicular monitoring in 81 patients of fertile age. These patients were referred for follicular monitoring in normal and stimulated cycles from infertility clinic. Patients' compliance and acceptance was excellent for TVS technique as compared to TAS technique. All the patients gives acceptance for TVS. Initial apprehension and hesitancy was noted in $15(18.51 \%)$ cases for TVS. That was easily overcome by proper explanation about the procedure and its better result. Two (2.46\%) patients had excessive apprehension leading to vaginismus at the attempt of introduction of TV probe. They were allowed to witness another patients being examined without any discomfort. This help in relieving of apprehension and we succeeded in TV scanning in both these patients. The overall resolution of ovarian and follicular anatomy like shape of follicles and antral adges was much better in TVS. All follicles were anechoic. With both method we found no difference in baseline size and TVS approach has higher accuracy in assessing number of follicles. There was no difference found number of dominant follicle in both method. There was significant difference in rate of growth of follicle in both ovary on comparing TVS and TAS, mean follicular growth was greater during transvaginal scanning. There was no significant difference in size of follicle in both ovary on comparing TVS and TAS. 
Besides resolution of the follicles, we also examined the relationship between the follicular size and detection rates of follicles by both the scanning methods. For follicles $>20 \mathrm{~mm}$ in size, no significant difference was noted in detection rate between TAS and TVS. However, in smaller follicles especially below $10 \mathrm{~mm}$ the TVS offered definite improvement in the detection rates. TVS even enabled us to detect and count follicles as small as 3-4 mm accurately. No TAS scanning could identify such small follicles and in all these cases the ovaries appeared as uniformly hypoechoic structures. It is important to record the number of follicle with their respective sizes in gonadotrophin stimulated cycles. Here again the TVS detection rate of follicles far exceeded TAS detection rate. The frequency of 5 or more growing follicles were detected in $88.7 \%$ in transvaginal scanning as compared to $12.9 \%$ in transabdominal scanning.

It is important to document serial follicular dynamics in normal as well as stimulated cycles in the management of infertility; TV scanning helps us to do that accurately. The sonologists can accurately guide the infertility specialist for timing administration of HCG, titrating dosage of gonadotrophin administration. Detection of occurrence of ovulation helps in timing of Intra uterine insemination (IUI). There is very good correlation of number of follicles detected with TVS and laparoscopic finding. Evidences also suggest that, transabdominal methods underestimates the number of follicles, than does TVS. TV ultrasound measurement of follicular size has lower intra and inter-observer variability. Other studies also demonstrated that TVS scanning methods have a higher degree of patients compliance and acceptance of TVS has already been confirmed in our study. This highlights the need to make use of this mode of investigation for wider indications.

We conclude that ultrasound play an important role in the monitoring of ovulation induction. The present role of ultrasound is to establish whether the ovary responds to gonadotropin stimulation, to ensure that the follicle has reached a optimum size, to determine the number of preovulatory follicles to inform better the patient about the possibilities of multiple pregnancies, to establish that ovulation has occurred after administration of hCG, and to document the presence or absence of OHS. With the introduction of GnRH agonists for pituitary down-regulation combined with gonadotropins for ovarian stimulation, It became clear that hormonal monitoring of the follicular maturation is not so crucial. Monitoring of stimulation can be done only by means of ultrasound, and in case of high suspicion for the development of OHSS, additional measures can be taken either before or after aspiration. Such a monitoring system will simplify the treatment and its cost. The situation is the same in patients who are undergoing conventional ovarian stimulation. In these patients, since we are bound to prevent multiple gestation, the final stage of ovulation will not be triggered if there will be more than 2 to 3 leading follicles. By keeping this limit, it is quite rare either to reach high levels of serum estradiol or to develop OHSS and thus measuring serum estrogen levels will not add significantly to efficacy or safety of the treatment. The above simplification of the monitoring protocols will increase both costeffectiveness and patients' convenience.

\section{Bibliography}

[1]. Andrcotti R F, Thompson G H, Janowitz W, Shapiro A, Zusmer N R. Endovaginal and transabdominal sonography of ovarian follicles. J Ultrasound Med 1989; 8: 555-560.

[2]. Ayush Goel, Praveen Jha; Follicular monitoring, Banglore ,India:2009. Available from: http://radiopaedia.org/articles/

[3]. Baerwald AR , Adams GP , Pierson RA: Characterization of Ovarian Follicular Wave Dynamics in Women., Canada: May 14, 2003.

[4]. Belaisch-Allart J, Dufetre C, Allart JP, De Monzon J. Comparison of transvaginal and transabdominal ultrasound for monitoring follicular development in an in-vitro fertilisation programe. Hum Reprod. 1991;6:688-9.

[5]. Berek JS: Basic principles, Berek \& Novak Gynecology $14^{\text {th }}$ ed. Lippincott Williams \& Wilkins, Philadelphia, United States of America:2007;pp.172-184.

[6]. Callen PW: Ultrasonographic Imaging in Infertility., Ultrasonography In Obstetrics And Gynecology: $5^{\text {th }}$ ed. Elsevier Saunders. Philadelphia, United States of America:2008; pp.986-1019.

[7]. Chamberlin G, Steer P: Anatomy of female Pelvis., Turnbull's Obstetrics $3^{\text {rd }}$ ed, Churchill Livingstone. Philadelphia, United States of America: 2006; pp.19.

[8]. Crum CP, Lee KL: Evaluation of cyclic endometrium and benign endometrial disorders, Diagnostic Gynecology And Obstetrics Pathology, Elsevier Saunders. Philadelphia, United States of America: 2006; pp.441-458.

[9]. Cunningham FG, Leveno KJ, Bloom SL, Hauth JC, Gilstrap III LC, Wenstorn KD: Implantation, Embryogenesis, and Placental Development. Williams Obstetrics $22^{\text {nd }}$ ed.Mc Graw Hill, United States of America: 2005;pp.39-43.

[10]. Debnath J et al. Follicular monitoring: Comparison of transabdominal and transvaginal sonography Medical Journal Armed Forces India. $2000 \mathrm{Jan}$; 56(1): 3-6.

[11]. Deichert I, Hackeloer B J, Dawne E. The sonographic and endocrinologic evaluation of the endometrium in the luteal phase. Hum Reprod 1986; 1: 219-222.

[12]. Dewbury K, Meire H, Cosgrove D, Farrant P: The ovaries and Infertility., Clinical ultrasound in Obstetrics and Gynaecology, Clinical ultrasound a comprehensive text , Second edition. Churchill livingstone. London, United Kingdom:2001;pp.63-76 and 99108 .

[13]. Farquhar CM, Birdsall M, Manning P, Mitchell IM. Transabdominal versus transvaginal ultrasound in the diagnosis of polycystic ovaries in a population of randomly selected women. Obstet Gynecol 64:546-552.

[14]. Ghazi Sedeq, Parez RM, Samira SSM, Shahla KA. A prospective comparison of transvaginal, transabdominal ultrasound and diagnostic curettage in the evaluation of endometrial pathology in Erbil. Zanco J. Med. Sci., Vol. 20, No. (1), 2016.

[15]. Mendelson EB, Bohm-Velez M Joseph N, Neiman HI, Gynaecologic Imaging : Comparison of transabdominal and transvaginal sonography. Radiology 1988; 166:321-4. 
[16]. Nahar Z , Islam AHMT, Akter H, Lovely NA: Comparison of Transabdominal and Transvaginal Sonography in Folicular Monitoring .Rajshahi Dhaka: 2008; Volume 21 Number 1.

[17]. Sadler TW: First week of development: Ovulation to implantation, Langman's Medical Embryology $12^{\text {th }}$ ed. Lippincott Williams \& Wilkins, a Wolters Kluwer business. Philadelphia, United States of America: 2014;pp.29.

[18]. Tsuda H, Kawabata M, Kawabata K, Yamamoto K, Hidaka A, Umesaki N. Comparison between transabdominal and transvaginal ultrasonography for identifying endometrial malignancies. Gynecol Obstet Invest. 1995;40(4):271-3. 